\title{
Atypical symptoms of gastro-esophageal reflux during pregnancy
}

\author{
Enrique Rey ${ }^{1}$, Fernando Rodríguez-Artalejo², Miguel Ángel Herraiz ${ }^{3}$, Ángel Álvarez-Sánchez ${ }^{1}$, \\ Manuel Escudero ${ }^{3}$ and Manuel Díaz-Rubio ${ }^{1}$

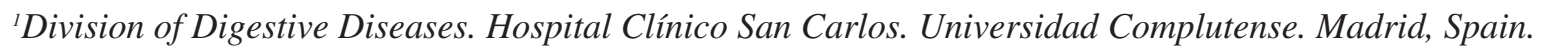 \\ ${ }^{2}$ Department of Preventive Medicine and Public Health. School of Medicine. Universidad Autónoma de Madrid. \\ CIBER of Epidemiology and Public Health (CIBERESP). ${ }^{3}$ Department of Obstetrics and Gynecology. \\ San Carlos University Teaching Hospital. Universidad Complutense. Madrid, Spain
}

\begin{abstract}
Background: pregnancy is associated with an increased incidence of heartburn. However, there is no information for other symptoms related to gastro-esophageal reflux (GOR).

Aim: to assess the prevalence of atypical symptoms of GOR during pregnancy, and to examine its association with typical GOR symptoms.

Methods: we report data for 263 women with a pregnancy of less than 12 weeks. They were interviewed at the end of each trimester of pregnancy and at 1-year post-partum, using the Gastro Esophageal Reflux Questionnaire (GERQ). In the first interview, information about symptoms in the year before pregnancy was also collected with GERQ.

Results: women suffered atypical GOR symptoms during pregnancy more frequently than in the year before: non-cardiac chest pain (NCCP) (9.1 vs. 1.9\%), dysphagia (12.6 vs. $2.3 \%$ ), globus (33.1 vs. $4.6 \%)$, cough (26.6 vs. 6.8\%), belching (66.2 vs. 19.4\%) and hiccups (19.0 vs. 8.4\%). Atypical GOR symptoms in pregnancy showed an association with suffering the same symptom before pregnancy and NCCP, globus, belching and hiccups with suffering typical GOR symptoms in the first trimester.

Conclusions: atypical GOR symptoms are highly prevalent in pregnancy, and are associated with atypical symptoms before pregnancy and with typical symptoms of GOR in the first
\end{abstract} trimester.

Key words: Gastro-esophageal reflux disease. Pregnancy. Chest pain. Cough. Dysphagia. Longitudinal study.

Rey Enrique, Rodríguez-Artalejo Fernando, Herraiz Miguel Ángel, Álvarez-Sánchez Ángel, Escudero Manuel, Díaz-Rubio Manuel. Atypical symptoms of gastro-esophageal reflux during pregnancy. Rev Esp Enferm Dig 2011; 103: 129-132.

Received: $14-06-10$.

Accepted: 14-10-10.

Correspondence: Enrique Rey. Servicio de Aparato Digestivo. Hospital Clínico San Carlos. c/ Martin Lagos s/n. 28040 Madrid, Spain.

e-mail: rey.enrique.spain@gmail.com

\section{INTRODUCTION}

Typical gastro-esophageal reflux (GOR) symptoms are frequent in western societies, with a prevalence ranging from 10 to $30 \%(1,2)$, and an incidence around $2-4 \%$ yearly (3). In addition to heartburn and acid regurgitation, other symptoms, collectively known as atypical symptoms, are potentially related to GOR; the support for including them under the spectrum of GOR is provided mostly by epidemiological cross-sectional studies (4), response to treatment and expert opinion (5).

Pregnancy promotes GOR and is associated with developing typical GOR symptoms (6-9), with an incidence of about $25 \%$ each trimester (10). However, there is no information on the prevalence of atypical symptoms during pregnancy (11), with the exception of one study, published only in abstract, which reported a high prevalence (12).

This paper assessed the prevalence of atypical symptoms of GOR during pregnancy, and examined their association with typical GOR symptoms.

\section{PARTICIPANTS AND METHODS}

Study methods and ascertainment of typical GOR symptoms have been reported elsewhere $(4,9)$. Briefly, we studied women, aged 18-45 years, with a pregnancy of less than 12 weeks from the late of last menses, who attended a obstetrics center in 2004. This center provides health care to the Latina District in Madrid (Spain), an area with a registered resident population of 244,261 persons in 2003. Women were followed over pregnancy and the post-partum year to evaluate symptoms of GOR.

Selection criteria for pregnant subjects were: not having been pregnant in the preceding year; having a telephone at home; having Spanish as their mother tongue; 
and not having undergone esophageal-gastric surgery or esophageal dilatation. Study participants gave their informed consent in writing. The study was formally approved by the Clinical Research Ethics Committee of the Hospital Clínico San Carlos in Madrid.

Information was obtained through telephone interviews conducted by trained professionals. Interviews were held at the end of each trimester of pregnancy and at 1-year post-partum. Data were collected using the Gastro-Esophageal Reflux Questionnaire (GERQ), which has been validated and adapted for telephone use in the Spanish population (13). At the end of the first trimester of pregnancy, the GERQ was administered twice: on the first occasion, the questions referred to the preceding year, and on the second, they referred to the 12 weeks following the commencement of pregnancy. When the GERQ was administered at the end of the second and third trimesters, the questions addressed the 12 weeks since the preceding interview. At the 1-year post-partum interview, questions addressed the year following delivery.

We defined typical GOR symptoms as the presence of weekly heartburn or acid regurgitation in the corresponding period, either one year before pregnancy, each trimester of pregnancy, or 1-year post-partum. Atypical symptoms were defined as follows: a) non-cardiac chest pain (NCCP), pain or discomfort in the chest different from heartburn, primarily non-abdominal, occurring at least weekly; since cardiac causes of chest pain were extremely uncommon in pregnant women (14), all chest pain reported was considered non-cardiac; b) dysphagia, sensation of food remaining lodged in the throat or chest, occurring at least weekly; c) belching, expulsion of air rising from stomach for at least $25 \%$ of the days; d) globus, sensation of having a lump or tightness in the throat when not swallowing for at least $25 \%$ of the days; e) cough, coughing more than 4 times per day for 4 days or more per week; and f) hiccups, where they were regarded by the woman as a problem.

The prevalence of each atypical symptom of GOR, along with its $95 \%$ confidence interval (CI), was calculated for the year before pregnancy, for each trimester of pregnancy, and for the post-partum year. Also, prevalence of the individual atypical symptoms was estimated according to the presence of typical GOR symptoms.

Multiple logistic regression models were used to examine factors independently associated with each atypical GOR symptom during pregnancy. Factors considered were the following: suffering the same symptom weekly in the preceding year, suffering typical GOR symptoms weekly during the year before pregnancy and during the first, second, and third trimester of pregnancy, body mass index before pregnancy, weight change during pregnan$\mathrm{cy}$, and other potential confounders of the study association, such as age, previous pregnancies, psychosomatic symptom score before pregnancy, marital status, education level, and employment status.
Statistical significance was set at two-sided $\mathrm{p}<0.05$. Statistical analyses were performed with SPSS v 13.0.

\section{RESULTS}

In all, 410 pregnant women were selected; of these, 365 were finally included, and $263(72.05 \%)$ completed the interviews in the three trimesters of pregnancy. In comparison with the 263 women who completed the three interviews during pregnancy, the 102 women who did not had a higher proportion of primigravidae $(61.2$ vs. $45.1 \%$; p < $0.05)$ and a higher psychosomatic symptom score (13.9 vs. $10.9 ; \mathrm{p}<0.05)$, with no other relevant differences.

Women suffered atypical GOR symptoms during pregnancy more frequently $(\mathrm{p}<0.05)$ than in the year before: NCCP (9.1 vs. $1.9 \%)$, dysphagia (12.6 vs. $2.3 \%)$, globus (33.1 vs. 4.6\%), cough (26.6 vs. 6.8\%), belching (66.2 vs. $19.4 \%)$ and hiccups (19.0 vs. 8.4\%). At 1-year post-partum, frequency of atypical GOR symptoms had decreased, to become similar to that in the year preceding pregnancy. Figure 1 disaggregates these data for each trimester of pregnancy.

Women with typical GOR symptoms any time during pregnancy suffered more frequently than those without from NCCP (12.8 vs. 3.7\%; p < 0.01), dysphagia (16.6vs. $6.5 \%$; $<<0.05)$, globus (43.6 vs. 17.8\%; p < 0.001 ), belching $(72.4 v s .57 .0 \% ; \mathrm{p}<0.01)$ and hiccups $(25.6 v s$. $9.3 \% ; \mathrm{p}<0.01)$. Comparisons with symptoms at 1 -year post-partum are not presented, because only 12 women reported typical GOR symptoms in the year following delivery. Table I expands this information by showing atypical symptoms of GOR for each trimester of pregnancy, according to typical GOR symptoms.

In the logistic regression model with adjustment for confounders, reporting NCCP during pregnancy was associated with frequent $\mathrm{NCCP}$ in the year before (odds ratio [OR] 32.1; 95\% confidence interval [CI] 2.6-458.1) and with frequent typical GOR symptoms in the first trimester (OR 4.3; 95\% CI 1.3-14.0). Dysphagia during pregnancy was only related to suffering dysphagia in the year before (OR 12.5; 95\% CI 1.2-131.3). Globus showed an association with frequent typical GOR symptoms in the first (OR 2.8; 95\% CI 1.4-5.7) and second trimesters (OR 2.1; 95\% CI 1.0-4.4) of pregnancy, and with a psychosomatic symptoms score $>8$ before pregnancy (OR 2.2; 95\% CI 1.1-4.4). Also, those with cough during pregnancy were more likely to have suffered cough in the year before (OR 9.8; 95\% CI 2.7-35.3) and to being multiparous (OR 2.5; 95\% CI 1.2-5.4). Belching was directly related to frequent typical GOR symptoms in the first trimester (OR 2.9; 95\% CI 1.2-6.9) and inversely to multi-parity (OR 0.4 ; $95 \%$ CI $0.2-0.8$ ). Lastly, experiencing hiccups was more frequent in those with hiccups in the preceding year (OR 4.9; 95\% CI 1.4-17.4) and with frequent typical GOR symptoms in the first trimester of pregnancy (OR6.0; 95\% CI 1.5-24.6). 

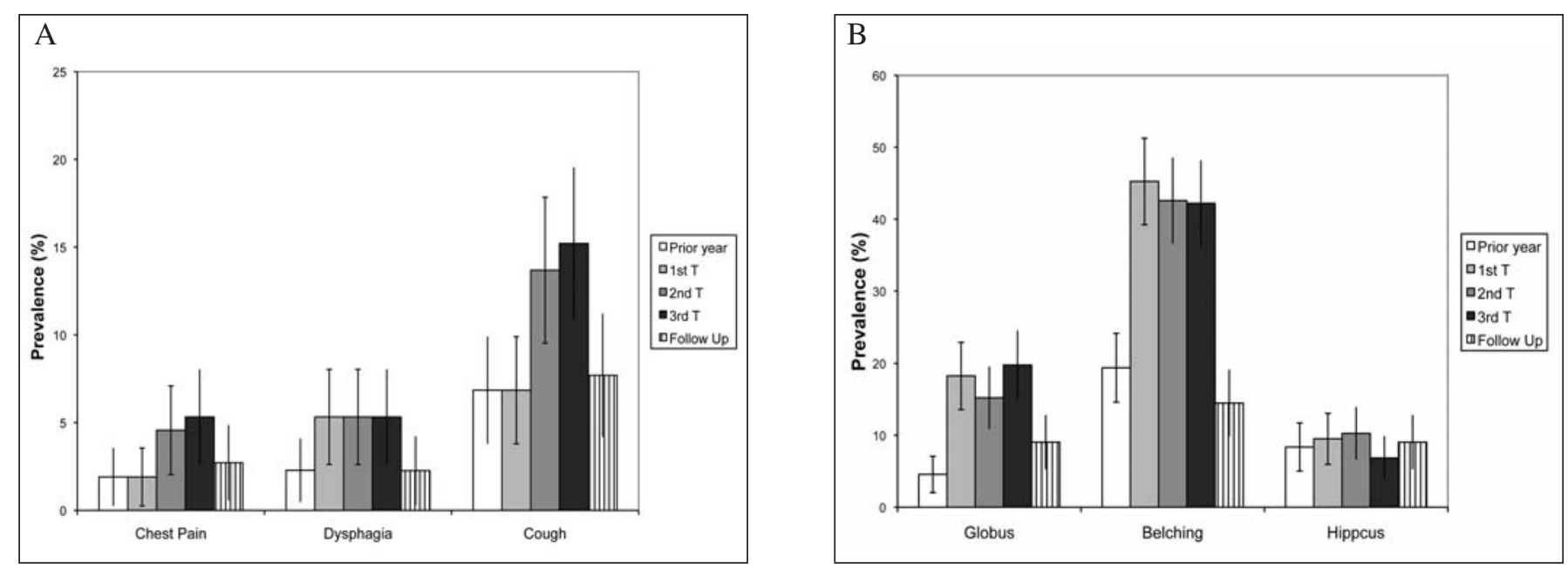

Fig. 1. Prevalence ( $95 \%$ confidence interval in vertical bars) of atypical symptoms of gastro-esophageal reflux in the year before pregnancy, in each trimester of pregnancy, and at 1-year post-partum. A. Dysphagia, chest pain and cough. B. Globus, belching and hiccups.

\section{DISCUSSION}

Our study provided a detailed description of the prevalence of atypical symptoms of GOR during pregnancy, and their associated risk factors. Prevalence of atypical symptoms of GOR is high during pregnancy, even higher than previously reported in abstract form (12). Around $10 \%$ of pregnant women referred weekly NCCP or dysphagia at any time during pregnancy, 30\% referred hiccups, close to $35 \%$ reported chronic cough or globus, and two thirds belching. Compared to preceding year, pregnancy presents with a prevalence of atypical GOR symptoms which is seven times higher for globus and cough, five times higher for dysphagia, four times for NCCP, three times for belching, and twice for hiccups. More- over, our results suggest that this increased frequency of symptoms is produced by pregnancy itself, because symptoms returned to baseline values at 1-year post-partum.

As regards the time-pattern of atypical GOR symptoms during pregnancy, our data suggest that two different patterns might exist. Frequency of NCCP and cough increased progressively over pregnancy, while frequency of dysphagia, globus and belching rose in the first trimester and hold during the rest of pregnancy. It also suggests that the mechanisms whereby GOR produces extraesophageal symptoms may differ across symptoms. Some symptoms, like chest pain, may require the persistence of reflux over time to occur while others may depend just on the effects of single reflux episodes.

Table I. Prevalence ( $95 \%$ confidence interval) of each atypical gastro-esophageal reflux (GOR) symptom in the year before pregnancy and in each trimester of pregnancy, according to typical GOR symptoms

\begin{tabular}{|c|c|c|c|c|c|}
\hline & & $\begin{array}{l}\text { Year before } \\
\text { pregnancy }\end{array}$ & $\begin{array}{l}\text { First trimester } \\
\text { of pregnancy }\end{array}$ & $\begin{array}{l}\text { Second trimester } \\
\text { of pregnancy }\end{array}$ & $\begin{array}{l}\text { Third trimester } \\
\text { of pregnancy }\end{array}$ \\
\hline \multirow[t]{2}{*}{ Chest pain } & No typical GORS & $1.6 \%(0.0-3.2)$ & $1.1 \%(-0.4-2.5)$ & $2.2 \%(0.1-4.3)$ & $2.6 \%(0.1-5.0)$ \\
\hline & Typical GORS & $5.3 \%(-4.8-15.3)$ & $4.1 \%(-0.5-8.7)$ & $10.0 \%(3.4-16.6)^{* *}$ & $9.3 \%(3.8-14.9)^{*}$ \\
\hline \multirow[t]{2}{*}{ Dysphagia } & No typical GORS & $2.0 \%(0.3-3.8)$ & $2.6 \%(0.4-4.9)$ & $3.8 \%(1.0-6.6)$ & $0.6 \%(-0.6-1.9)$ \\
\hline & Typical GORS & $5.3 \%(-4.8-15.3)$ & $12.3 \%(4.8-19.9)^{* *}$ & $8.8 \%(2.6-14.9)$ & $12.1 \%(6.0-18.3)^{* *}$ \\
\hline \multirow[t]{2}{*}{ Globus } & No typical GORS & $3.3 \%(1.0-5.5)$ & $12.1 \%(7.5-16.7)$ & $8.2 \%(4.2-12.2)$ & $12.8 \%(7.6-18.1)$ \\
\hline & Typical GORS & $21.1 \%(2.7-39.4)^{\star *}$ & $34.2 \%(23.4-45.1)^{\star *}$ & $31.3 \%(21.1-41.4) * *$ & $29.9 \%(21.2-38.6)^{* *}$ \\
\hline \multirow[t]{2}{*}{ Cough } & No typical GORS & $7.0 \%(3.8-10.2)$ & $6.3 \%(2.9-9.8)$ & $9.3 \%(5.1-13.5)$ & $13.5 \%(8.1-18.8)$ \\
\hline & Typical GORS & $5.3 \%(-4.8-15.3)$ & $8.2 \%(1.9-14.5)$ & $23.8 \%(14.4-33.1)^{* *}$ & $17.8 \%(10.5-25.0)$ \\
\hline \multirow[t]{2}{*}{ Belching } & No typical GORS & $18.0 \%(13.2-22.9)$ & $38.4 \%(31.5-45.3)$ & $38.8 \%(31.7-45.9)$ & $33.3 \%(25.9-40.7)$ \\
\hline & Typical GORS & $36.8 \%(15.1-58.6)$ & $63.0 \%(51.9-74.1)^{* *}$ & $51.3 \%(40.3-62.2)$ & $55.1 \%(45.7-64.6)^{* *}$ \\
\hline \multirow[t]{2}{*}{ Hiccups } & No typical GORS & $7.0 \%(3.8-10.2)$ & $7.9 \%(4.1-11.7)$ & $9.3 \%(5.1-13.5)$ & $3.8 \%(0.8-6.9)$ \\
\hline & Typical GORS & $26.3 \%(6.5-46.1)$ & $13.7 \%(5.8-21.6)$ & $12.5 \%(5.2-19.8)$ & $11.2 \%(5.2-17.2)^{*}$ \\
\hline
\end{tabular}

${ }^{*} \mathrm{p}<0.05 ;{ }^{* *} \mathrm{p}<0.01$ (Fischer's test). GORS: GOR symptoms. 
The association of atypical with typical GOR symptoms was clearly evident, regardless of whether it was examined globally (any time in pregnancy) or by trimester. Time-patterns commented above were even more evident when data were analyzed by trimester and stratified by the typical GOR symptoms. For example, prevalence of NCCP and cough increased specially in the second and third trimesters in women with typical GOR symptoms. However, prevalence of dysphagia, globus and belching was higher from the beginning of pregnancy in women with typical symptoms of GOR. Of note is the increase in prevalence of globus and belching among women with no typical symptoms, suggesting that there is an additional promoting factor.

Two main risk factors were found to be associated with atypical GOR symptoms. First, suffering the same symptom before pregnancy is clearly a risk factor for each symptom during pregnancy, except globus and belching. It suggests that the symptomatic expression of GOR in each subject is quite specific, and tends to suffer the same atypical symptoms over time. Secondly, suffering typical GOR symptoms during the first trimester is associated with atypical symptoms, except dysphagia and cough. That association may lead to the hypothesis that most atypical symptoms are more likely if there is a persistence of reflux over time.

Some methodological aspects of the study warrant mention. First, data were self-reported during pregnancy and one year afterwards. This could increase prevalence of GOR symptoms in the year before pregnancy through erroneous recall. Nevertheless pregnancy is an organic condition of a sufficiently singular nature to enable subjects to distinguish whether or not symptoms preceded the pregnancy; indeed, the prevalence of atypical symptoms in the year before pregnancy was much lower than during pregnancy. Second, our analyses were adjusted for the most important confounders. Although it cannot totally rule-out a certain residual confounding, it is likely to be small because the association between atypical and typical GOR symptoms was observed both in the crude and the adjusted analyses. Third, near $30 \%$ of pregnant women were lost to follow-up during pregnancy, mostly due to spontaneous abortion and inability to interview them in the narrow window established by protocol. Although we cannot exclude a response bias, there were minor differences between those followed and those lost; also, differences were observed only for variables (proportion of primigravidae and psychosomatic symptoms score) that were not found to be related to GOR symptoms at any time during pregnancy in previous analyses (4). Finally, data were collected through telephone interviews, and in Spain the response rate has been shown to be better with telephone than with direct person-to-person interviews (2). Finally, we acknowledge that the optimum would have been to start the study before pregnancy, but it is not feasible in real-life practice.

In conclusion, pregnancy is associated with a remarkable rise in the prevalence of atypical symptoms of GOR, which return to baseline values in the year following deliv- ery. The main factors associated with atypical GOR symptoms during pregnancy are atypical symptoms before pregnancy and typical symptoms of GOR in the first trimester. Whether treatment with PPI is effective for atypical symptoms during pregnancy is unknown, but our data show a clear longitudinal relationship between these symptoms and typical reflux symptoms, suggesting that it may be worthy in severe cases. Also, prognostic information may be provided to pregnant women: suffering reflux in the first trimester predicts a higher risk of other reflux symptoms during pregnancy, and secondly the disappearance of symptoms after delivery may be anticipated in most cases.

\section{ACKNOWLEDGMENTS}

We thank the obstetricians and obstetric nurses of the Health Center "Avenida de Portugal" for their collaboration in the recruitment of study participants.

\section{REFERENCES}

1. Dent J, El-Serag HB, Wallander MA, Johansson S. Epidemiology of gastro-oesophageal reflux disease: a systematic review. Gut 2005;54: 710-7.

2. Díaz-Rubio M, Moreno-Elola-Olaso C, Rey E, Locke GR, III, Rodríguez-Artalejo F. Symptoms of gastro-oesophageal reflux: prevalence, severity, duration and associated factors in a Spanish population. Aliment Pharmacol Ther 2004;19:95-105.

3. Rey E, Choung RS, Schleck CD, Zinsmeister AR, Locke GR, III, Talley NJ. Onset and risk factors for fecal incontinence in a US community. Am J Gastroenterol 2010;105:412-9.

4. Rey E, Elola-Olaso CM, Rodríguez-Artalejo F, Locke GR, III, DíazRubio M. Prevalence of atypical symptoms and their association with typical symptoms of gastroesophageal reflux in Spain. Eur J Gastroenterol Hepatol 2006;18:969-75.

5. Vakil N, van Zanten SV, Kahrilas P, Dent J, Jones R. The Montreal definition and classification of gastroesophageal reflux disease: a global evidence-based consensus. Am J Gastroenterol 2006;101:1900-20.

6. Bassey OO. Pregnancy heartburn in Nigerians and Caucasians with theories about aetiology based on manometric recordings from the oesophagus and stomach. Br J Obstet Gynaecol 1977;84:439-43.

7. Isolauri J, Laippala P. Prevalence of symptoms suggestive of gastrooesophageal reflux disease in an adult population. Ann Med 1995;27: 67-70.

8. Marrero JM, Goggin PM, de Caestecker JS, Pearce JM, Maxwell JD. Determinants of pregnancy heartburn. Br J Obstet Gynaecol 1992; 99:731-4.

9. Tosal HB, Richart MM, Luque PM, Gutierrez L, Pastor GR, Cabrero GJ, Reig FA. Gastrointestinal signs and symptoms during pregnancy and postpartum in a sample of Spanish women]. Aten Primaria 2001; 28:53-8.

10. Rey E, Rodriguez-Artalejo F, Herraiz MA, Sanchez P, AlvarezSanchez A, Escudero M, Diaz-Rubio M. Gastroesophageal reflux symptoms during and after pregnancy: a longitudinal study. Am J Gastroenterol 2007;102:2395-400.

11. Richter JE. Gastroesophageal reflux disease during pregnancy. Gastroenterol Clin North Am 2003;32:235-61.

12. Thomas MR, Cutler A, Winslow C. Atypical presentation of gastroesophageal of gastroesophageal reflux disease in pregnancy. Am J Gastroenterol 97 (Supl.) ed. 2002:S27-S28.

13. Moreno Elola-Olaso C, Rey E, Rodríguez-Artalejo F, Locke GR, III, Diaz-Rubio M. Adaptation and validation of a gastroesophageal reflux questionnaire for use on a Spanish population. Rev Esp Enferm Dig 2002;94:745-58

14. Fayomi O, Nazar R. Acute myocardial infarction in pregnancy: a case report and subject review. Emerg Med J 2007;24:800-1. 